\title{
Dan Zeman
}

\section{The use of the Binding Argument in the debate about location}

\begin{abstract}
In this paper I inquire into the methodological status of one of the arguments that have figured prominently in contemporary debates about the semantics of a variety of expressions, the so-called "Binding Argument". My inquiry is limited to the case of meteorological sentences like "It is raining", but my conclusion can be extended to other types of sentences as well. Following Jason Stanley, I distinguish between three interpretations of the argument. My focus is on the third, weakest interpretation, according to which postulating variables for locations in the logical form of meteorological sentences is the best available explanation of the binding phenomena that such sentences give rise to. My aim is to show that even in this weak interpretation, the argument cannot be reasonably taken to hold. I accomplish this by showcasing several alternative ways to account for the binding data that have not been, as of yet, ruled out as flawed.
\end{abstract}

In recent debates about the semantics of various types of expressions, one often encounters a particular argument purporting to show that certain sentences have a variable of a certain kind in their logical form. ${ }^{1}$ This is the "Binding Argument": it proceeds from the observation that complex sentences containing a quantificational phrase operating on simple sentences (the target sentences) have a bound reading, to the conclusion (obtained by employing prima facie plausible claims about the connection between semantics and syntax) that the target sentences have a certain variable in their logical form. The argument has been used against more than one view on the market and has been applied to a variety of expressions. Due to its widespread acceptance, from a methodological point of view the Binding Argument seemed to be a safe bet.

In this paper I will be concerned with the use of the Binding Argument in the debate over the semantics of meteorological sentences like "It is raining". To be more precise, I will be concerned with the methodological efficacy of the argument under one of its interpretations: namely, that according to which the postulation of certain variables in the logical form of the target sentences is the best

1 "Logical form" will be understood in this paper as referring to the level of syntactic representation free of ambiguities that constitutes the input to semantics. 
explanation of the binding data proponents of the argument rely on. ${ }^{2}$ According to such an interpretation, the postulation of the relevant variables is the best explanation if i) explains the binding data; ii) there are no other accounts of the data that are better situated to do so. My aim is to show that, contrary to what its proponents claim, the Binding Argument under this interpretation is ineffective, for the simple reason that there is a number of alternative ways to account for the binding data that don't postulate variables in the logical form of the target sentences and which have not been ruled out as faulty. I will thus present, in quite significant detail, several such alternative ways. The claim is not that such ways are better situated than the variables-postulation strategy to explain the binding data. Rather, it is that without a thorough examination of those ways and a careful comparison between them and the explanation consisting in postulation of variables in the logical form of the target sentences the Binding Argument (in the intended interpretation) cannot be reasonably taken to hold.

The paper is structured as follows. I start with introducing the argument by means of an example taken from the debate between two mainstream views on the semantics of meteorological sentences and then offer a more general characterization of it as an argument-schema. In section 2 I present three interpretations of the argument and make clear which one I'm concerned with in this paper. In the same section I show that the third, weaker interpretation of the argument is consistent with criticisms leveled against the argument under the first two interpretations. Section 3 is then dedicated to the presentation of five alternative ways to account for the bound readings of complex sentences containing sentences like "It is raining" without postulating variables for locations in their logical form. In the last section I restate the main claims of the paper and their implications for the semantics of meteorological sentences.

\section{The Binding Argument: an example and an argument-schema}

To get a feel of how the Binding Argument is supposed to work, let's start with a concrete example of how it has been used in one of the most vivid

2 This interpretation of the Binding Argument has been offered by one of the proponents of the argument, Stanley (2007, chapter 1). Other authors dealing with the argument take it to have such an interpretation (see, for example, Sennet (2008: 143)). Regarding the Binding Argument itself, its origin can be traced back to Chomsky (1957). 
debates in recent philosophy of language. When it comes to the semantics of meteorological sentences like 'It is raining', the two major views found in the literature can be subsumed under the labels truth-conditional semantics and truth-conditional pragmatics. The bone of contention between these two general views is whether such sentences have variables for locations in their logical form or not. Without going into much detail, truth-conditional semantics is the view (or, rather, a cluster of views) characterized by the claim that "any contextual effect on truth-conditions that is not traceable to an indexical, pronoun, or demonstrative (...) must be traceable to a structural position occupied by a variable” (Stanley 2007: 39). In contrast, truth-conditional pragmatics is the view (or, rather, a cluster of views) according to which the provision of elements in the content of utterances is done via optional pragmatic processes like free enrichment rather than by mandatory ones such as saturating a variable in the logical form (Recanati 2002, 2004 being the most prominent defender of the view). Now, the Binding Argument has been used to argue against truth-conditional pragmatics by adepts of a truth-conditional semantic view about meteorological sentences. Thus, Stanley (2007, chapter 1) notes that sentence

(1) Every time John lights a cigarette, it rains

has a reading according to which every time John lights a cigarette at time $t$, it rains at time $t$ at the location in which John lights a cigarette. The location of rain in (1) is thus bound by the quantifier phrase "every time John lights a cigarette". With this reading of (1) in mind, and using the regimentation of a similar argument given by Recanati, the instance of the Binding Argument against truthconditional pragmatics about locations could be put as follows:

1L. According to truth-conditional pragmatics, there is no variable for the location of rain in the logical form of the sentence 'It is raining'.

2L. In (1), binding occurs: the location of rain varies with the values introduced by the quantifier 'every time John lights a cigarette'.

3L. There is no binding without a bindable variable in the logical form.

4L. Therefore, there is a variable for the location of rain in the logical form of the sentence 'It is raining'.

5L. Therefore, truth-conditional pragmatics is mistaken. (Adapted from Recanati 2002: 328-9).

The same reasoning can be applied to argue against different views about the semantics of "It is raining". Relativism about such sentences is a case in point. Relativism is the view (or, rather a cluster of views) according to which the location is contributed by the circumstances in which utterances of "It is raining" 
are evaluated for truth. ${ }^{3}$ Assuming that a location cannot be provided both by a variable in the logical form of a sentence and by the circumstances with respect to which utterances of that sentence are evaluated for truth ${ }^{4}$, the Binding Argument can be used to refute relativism by showing that a location has to be provided by the former. On the other hand, there are many other types of sentences besides meteorological ones in connection to which various instances of the Binding Argument have been employed. Thus, drawing on the work of Barbara Partee and Kai von Fintel, Stanley (2007, chapters 1 and 6, for example) uses instances of the Binding Argument to argue against truth-conditional pragmatic views about a wide range of expressions, including gradable adjectives, quantifier phrases, relational expressions such as 'local', ‘enemy', etc. Schaffer (2011) uses instances of the argument to argue both against relativism about predicates of personal taste and epistemic modals. This shows the argument's versatility: it can be used to argue against several views about the semantics of various types of sentences. This, in turn, might explain the argument's recent popularity in semantics.

I mentioned above that my focus in this paper is on meteorological sentences, but the replies to the Binding Argument I will investigate below can easily be extended to other types of sentences. Because of this, it would be useful to have a more general rendering of the argument, one that abstracts away from particular instances found in the literature. Building on the regimentation provided by Recanati, the Binding Argument can be cast in the form of the following argument-schema: ${ }^{5}$

(1) According to position $\mathbf{P}$, sentence $s$ doesn't have a variable for parameter $\pi$ in its logical form.

(2) Sentences of the form $Q(s)$ have a bound reading where parameter $\pi$ is bound by the quantifier phrase $Q$.

(3) There is no binding without a bindable variable in the logical form.

(4) Therefore, sentence $s$ has a variable for parameter $\pi$ in its logical form.

(5) Therefore, position $\mathbf{P}$ is mistaken.

3 Relativism about meteorological sentences is not a very popular view, although see Lewis (1980) for an early relativist (in the sense used here) position. Kaplan (1989) also toyed with the idea of introducing locations in the circumstances of evaluation. For a contemporary defender of the view, see Recanati (2007).

4 A principle Recanati (2007: 34) dubs "Distribution". For a discussion of this principle in the context of employing the Binding Argument against relativism, see Zeman (2015).

5 A similar schema, with roughly the same premises, can be extracted from Sennet's (2008) discussion of the argument. 
One problem with this schema, and thus one that transfers to all its instances, is that, as it stands, it is invalid: the step from premises 1-3 to conclusion 4 is not mandated. Strictly speaking, what follows from the first three premises is the intermediary conclusion (IC):

(IC) Therefore, sentence $Q(s)$ has a variable for parameter $\pi$ in its logical form.

In order to get conclusion 4, we need a bridging principle relating sentences $s$ and $Q(s)$ in such a way as to make sure that the existence of a variable in the logical form of $Q(s)$ guarantees the existence of a variable of the same type in the logical form of $s$. Recanati (2002) provides such a principle. Discussing the instance of the argument presented above, Recanati notes that it is missing an additional premise and claims that ignorance of that fact has lead Stanley to commit "the binding fallacy". He claims that the following supplementary premise (SUP) is what the proponents of the relevant instance of the argument need in order for it to go through:

(SUP) In (1), the sentence on which the quantifier 'every time John lights a cigarette' operates is the very sentence 'It is raining' which can also be uttered in isolation. (Adapted from Recanati 2002: 329)

Recanati speaks about the same sentence here, but what he means is really a representation of the sentence at a deeper level than the linguistic surface. For my purposes here, I will take that deeper level to be the sentence's logical form. Thus, extrapolating from Recanati's suggestion, we arrive at the following bridging principle (BP):

(BP) The logical form of sentence $s$ is the same in $Q(s)$ as when $s$ appears in isolation. ${ }^{6}$

Using this principle and the intermediary conclusion above, we finally get the following valid argument-schema:

(1) According to position $\mathbf{P}$, sentence $s$ doesn't have a variable for parameter $\pi$ in its logical form.

(2) Sentences of the form $Q(s)$ have a bound reading where parameter $\pi$ is bound by the quantifier phrase $Q$.

(3) There is no binding without a bindable variable in the logical form.

6 With a similar purpose in mind, Sennet offers what he calls "The Semantic Innocence Assumption" (2008: 143). The differences between (BP) and Sennet's proposal are minimal. 
(IC). Therefore, sentence $Q(s)$ has a variable for parameter $\pi$ in its logical form (BP). The logical form of sentence $s$ is the same in $Q(s)$ as when $s$ appears in isolation.

(4) Therefore, sentence $s$ has a variable for parameter $\pi$ in its logical form.

(5) Therefore, position $\mathbf{P}$ is mistaken.

\section{Three interpretations of the Binding Argument}

With the argument in good standing, we can now inquire into its methodological status. There are at least three interpretations of the argument, each bestowing upon it a different methodological strength. The three interpretations alluded to have been provided by Stanley himself, when answering various criticisms of the Binding Argument. In what follows I will briefly discuss each of them, as well as the main objection pertaining to each interpretation.

According to the first interpretation, the argument "establishes the existence of covert structure, on pain of ungrammaticality due to vacuous binding” (Stanley 2007: 214). On the second, "a bound reading of a sentence is evidence for syntactic structure, since bound readings are the semantic effect of a syntactic process (...). [C] ertain kinds of semantic phenomena (for example, bound readings (...)) have ultimately a syntactic explanation.” (Stanley 2007: 214). Finally, “[o]n the third (and weakest) interpretation of the binding argument, it is an inference to the best explanation. By postulating a covert variable, one can account for the bound reading, and there is no other satisfactory way to account for it" (Stanley 2007: 214, my emphasis). While in its first and second interpretations the argument leads more or less directly to the postulation of variables (the bound readings establish and, respectively, are evidence for the presence of variables), this does not hold with its third. Under this interpretation the phenomena by themselves don't lead directly to the postulation of variables; this result is rather achieved by accounting for the data and by showing that there are no alternative, equally plausible, explanations of it. In this sense the third interpretation of the argument is weaker than the first two.

As mentioned from the outset, it is this third way of interpreting the Binding Argument that I will focus on in what follows. My aim is to show that, despite Stanley's (2007, chapter 6) suggestion that for certain sentences the postulation of variables in logical form is the best explanation for bounds readings, this is not the case with meteorological sentences like 'It is raining'. The reason the Binding Argument in its third interpretation doesn't succeed in forcing one to postulate variables for locations in the logical form of such sentences is that there are many alternative 
ways to account for the bound readings. Some of those alternative ways have been criticized; others have not even been addressed. However, the point is that without showing that all the alternative ways of handling the data are implausible or less plausible than the postulation of variables for locations in logical form, the Binding Argument in the interpretation considered here cannot be used to that effect.

Before moving to the detailed overview of some of the alternative ways of accounting for the data, let me first mention two common objections raised against the argument in its stronger (first and second) interpretations that will also help situate the present paper with respect to other criticisms of the Binding Argument. Thus, one early objection to the Binding Argument has been that it overgenerates massively. Perhaps the most striking illustration of this point is that offered by Cappelen and Lepore (2002), who, starting from the bound reading of the sentence

(2) Everywhere I go, $2+2=4$

and following the logic of the Binding Argument, arrive at the absurd conclusion that we would need to postulate a variable for locations in the logical form of sentences like " $2+2=4$ ". More generally, given that virtually any sentence could be embedded in a more complex sentence that has a bound reading, we would end up postulating many more variables in the syntax than seems plausible.

Now, Stanley (2007, chapter 6) has initially replied to this objection by distinguishing between the three interpretations of the Binding Argument mentioned above and by relegating Cappelen and Lepore's worries to the first of these. I'm not sure that the point made by Cappelen and Lepore had anything essentially to do with ungrammaticality and thus whether Stanley's move avoids their objection. However, it is also not clear that their objection is fatal. As both Stanley (2007, chapter 6) and Cohen and Rickless (2007) show, there are ways for the proponent of the Binding Argument to avoid the charge of overgeneration. Cohen and Rickless, for example, note a crucial disanalogy between Cappelen and Lepore's example and (1), claiming that the crucial feature underlying binding phenomena (namely, that the interpretation of that embedded sentence should systematically be affected by the quantifier) is present in the latter but absent in the former. Be that as it may, the point that I want to stress here is that even if Cappelen and Lepore's objection shows that the Binding Argument in its first (or, if Stanley's classification of their objection is wrong, its second) interpretation is flawed, this does not mean that the argument in its third interpretation is flawed too. In other words, it is still possible that, despite overgeneration problems, the postulation of variables for locations in the logical form of sentences is the best available explanation for the phenomena.

The main criticism of the Binding Argument in its second, strongest interpretation is that the prima facie plausible principle the proponent of the argument 
uses to move from the existence of bound readings to the postulation of variables in the logical form of the target sentences is, in fact, not well supported. What I have in mind here is premise 3 in argument-schema provided above, "there is no binding without a bindable variable in the logical form". This is a claim about the connection between semantic binding (which is what the intuitive bound readings put forward by the proponents of the Binding Argument are supposed to illustrate) and syntactic binding (which is a highly constrained, well regimented syntactic phenomenon). ${ }^{7}$ Many authors have denied premise 3 or similar claims (e.g., Carston 2002; Breheny 2003; Neale 2007; Collins 2007 - as well as most proponents of the answers to the Binding Argument that will be presented in the next section). Here, however, I will focus on one of the more recent and most substantial denials in the literature, the one found in Pupa and Troseth (2011). They object to what they call "coincidence": the claim that there is a perfect overlap between semantic binding and syntactic binding. ${ }^{8}$ Stressing the point that the proponents of the Binding Argument incur a very heavy theoretical burden of a syntactic nature, they show that there are clear cases in which there is semantic binding without there being syntactic binding, for reasons having to do with the impossibility of moving for certain expressions or with lack of suitable expressions that should be c-commanded. ${ }^{9}$

Now, Pupa and Troseth's criticism has focused on the case of domain restriction, but the same criticism might apply to the case of meteorological sentences as well. Indeed, they claim that "there's little hope for [variables-postulating approaches] to similar phenomena such as the (...) spatial variability of weather verbs" (Pupa and Troseth 2011: 207). To be sure, no such evidence has been given. But, as before, what I want to point out here is that even if such evidence were to be given, this would still be compatible with the third, weaker interpretation of the Binding Argument. In other words, even if the binding data don't provide conclusive evidence for the postulation of variables for locations in the logical form of meteorological sentences, it is still possible that such a postulation is the best available explanation for the phenomena. What matters according to the third interpretation of the argument is whether there are better ways to explain the phenomena than the variable-postulating approach. Pupa and Troseth have

\footnotetext{
7 To get the required reading, 'binding' in premise 3 has to be understood as semantic binding, while 'bindable' as syntactic binding.

8 Coincidence is assumed by Stanley (2007, chapter 1).

9 C-command is a crucial notion underlying the study of syntactic binding. Roughly, node $\mathrm{N}$ dominates node $\mathrm{M}$ if $\mathrm{N}$ is above $\mathrm{M}$ in the syntactic tree and there is a path from $\mathrm{N}$ to $\mathrm{M}$ that moves only downwards.
} 
shown that there are reasons to be dissatisfied with the argument in its second interpretation, but this does not mean that, in its third interpretation, the argument is useless. What does show its uselessness, however, is the following section.

\section{Alternative accounts of the binding data}

Several replies have been explicitly given, and others could easily be extracted from the literature, to various instances of the Binding Argument - including the one I have focused on here. Perhaps the most radical answer consists in rejecting the argument by eschewing variables altogether. In variable-free frameworks such as that developed in Jacobson (1999), for example, the Binding Argument doesn't make much sense. Challenging as they are, however, I will leave such frameworks aside in this paper. A more common answer is to accept variables, but to show that the data to be accounted for (such as the bound readings of sentences like (1)) can be handled in a framework that doesn't require the postulation of variables (of the relevant kind) in the logical form of the target sentences.

From the five ways to account for the data I have chosen to survey below, the first four rest on the idea of accounting for the bound readings of sentences like (1) by replacing quantification over locations with quantification over more encompassing entities, such as events, situations, contexts or indices. There are two ways to implement this idea. The first is to replace the variables for locations in the object language with variables for more encompassing entities in the object language itself. This way of implementing the basic idea is close to the spirit of Stanley's proposal, if not to its letter, in holding on to the claim that binding has to be accounted for by the postulation of variables in the logical form - albeit not of the same kind as the ones the conclusion of the Binding Argument would compel us to postulate. ${ }^{10}$ To illustrate this way to account for the data, I will present a view that uses event variables instead of location variables, the "Event Analysis" proposed by Cappelen and Hawthorne (2007), and a similar view that could be

10 This answer seems to be compatible with the existence of instances of the Binding Argument that argue for the postulation of variables for those more encompassing entities themselves. However, I take it that the dialectical power of the Binding Argument resides in its capacity to argue against views that deny that we need variables for very specific parameters (such as locations) in the logical form of the target sentences. In any case, the existence of alternatives in which no variable of any kind needs to be postulated at the object level is enough to support my point. 
extracted from Elbourne's (2005, 2008) work that uses situation variables. For each view presented, I will show how exactly the derivation of the conclusion of the Binding Argument is blocked.

The second way to implement the idea behind the type of approach mentioned is to replace the variables for locations in the object language with variables for the more encompassing entities in the metalanguage. Here, the departure from the proponents of the Binding Argument is clearer. To illustrate, I will present two versions of this way to account for the data: Pagin's (2005) proposal to use quantification over contexts and Lasersohn's (2008) quantification over indices view. As before, for each view presented, I will show how exactly the derivation of the conclusion of the Binding Argument is blocked.

The fifth proposal that I will present is more peculiar. It differs from the others in granting all the premises of the argument, while at the same time blocking its conclusion. This view has been proposed by Recanati's (2002, 2004) and consists in appeal to variadic operators, a device he introduced precisely with the aim of responding to the instance of the Binding Argument directed against a truthconditional pragmatic view of meteorological sentences.

The five alternative ways to account for the binding data just mentioned are underpinned by different commitments and their proponents are following more or less determinate agendas. (For example, Recanati's employment of variadic operators is directly connected with his general truth-conditional pragmatic view and he takes the avoidance of the conclusion of the Binding Argument to support that view.) For our purposes here, however, the differences between those approaches can be ignored. What is important is that under the views to be scrutinized the binding data are accounted for and that the instance of the Binding Argument presented in section 1 is blocked.

\subsection{Quantifying over events}

The alternative to postulating variables for locations in the logical form of meteorological sentences that appeals to variables for events has been proposed by Cappelen and Hawthorne (2007). Their view, which they call the "Event Analysis", is meant to be an alternative to Stanley's (2007, chapter 1) treatment of sentences such as (1), and it consists in adopting a Davidsonian event semantics (see for example Davidson (1967)) in combination with the idea that there are domain restrictions attached to certain phrases. In this case, the relevant phrases to attach domain restrictions to are verbs, which following the Davidsonian analysis should be conceived of as predicates of events. In this framework, binding phenomena are understood in terms of domain restrictions of events where the restrictor on 
events is bound by a higher quantifier. In the case of (1), the relevant type of higher quantifier is a temporal quantifier. Let me illustrate the view with an example given by the authors themselves. The bound reading of sentence

(3) Every time Sam goes to the park, Nina is walking her dog,

according to which every time Sam goes to the park Nina is walking her dog in the park where Sam goes to, is represented in the Event Analysis as

(4) For all times $t$, if there is an event $e_{1}$ that is a going to the park by Sam at $t$, there is an event $e_{2}$ that is a walking ${ }_{f(t)}$ of a dog by Nina,

where $t$ is the time of event $e_{1}$ (quantified over), while $f(t)$ is a function from times $t$ to the set of events that take place at $t$ in the park where Sam goes. Walking ${ }_{f(t)}$ is thus the set of events arrived at by intersecting the set of events that take place at $t$ in the park where Sam goes with the set of events of walking - that is, the set of events of walking that take place at $t$ in the park where Sam goes.

The problematic sentence (1) is represented in the event analysis as

(5) For every time $t$, if there is an event $e_{1}$ that is a lightning of a cigarette by John at $t$, then there is an event $e_{2}$ that is a raining ${ }_{f(t)}$,

where $t$ is the time of the event $e_{1}$ (quantified over), while $f(t)$ is a function from times $t$ to the set of events that take place at $t$ in the location where John lights a cigarette. Raining $f_{f(t)}$ is thus the set of events obtained by intersecting the set of events that take place at $t$ in the location where John lights a cigarette with the set of events of raining - that is, the set of events of raining that take place at $t$ in the location where John lights a cigarette.

How does this treatment help with blocking the Binding Argument? If (5) is the representation of (1) in the Event Analysis, then (IC) doesn't hold - there is no variable for locations in (5). But if it doesn't hold, then it means that either (IC) doesn't follow from the premises, and thus the argument is not valid, or one of the premises needed for its derivation is rejected. So, which is the case? Actually, both hold. Premise 3, the principle that there is no binding without a bindable variable in the logical form, is ambiguous between a reading according to which there is no binding without a bindable variable in the logical form simpliciter, and a reading according to which there is no binding without a bindable variable of the same kind as the bound parameter (here, location) in the logical form. On the first of this readings premise 3 holds under the Event Analysis (since we have a variable for events in (5)), but (IC) doesn't follow; on the second reading, the premise is rejected (since there is no location variable in (5)), but the location of rain is nevertheless bound. This means that in each situation the intermediary conclusion (IC) cannot be derived, and thus the Binding Argument is blocked. 


\subsection{Quantifying over situations}

A related way to account for the bound reading of (1) is one that replaces the postulation of variables for locations in the logical form of meteorological sentences with the postulation of variables for situations. Although not explicitly proposed for the case of locations, such a view could be extracted from Elbourne's (2005) treatment of donkey sentences. In contrast to whole worlds, situations are spatially and temporally limited parts of worlds. However, in order to get around well-known objections to the traditional notion of situation ${ }^{11}$, a more fine-grained notion has been introduced: that of a minimal situation. A minimal situation $s$ such that $p$ is a situation that contains just enough individuals, relations and properties to make $p$ true. Also, we need the part-of relation (symbolized $\leq$ ) to which situations are subject to: a situation $s$ is part of a situation $s^{\prime}$ if and only if $s$ ' contains all the individuals, properties and relations that $s$ does (and possibly some more). Now, in Elbourne's framework, which uses minimal situations, sentence

(6) If a farmer owns a donkey, he always beats the donkey and the priest beats the donkey too

is represented as

(7) $\lambda s_{1}$. for every minimal situation $s_{2}$ such that $s_{2} \leq s_{1}$ and there is an individual $x$ such that $x$ is a farmer in $s_{2}$ and there is an individual $y$ such that $y$ is a donkey in $s_{2}$ and $x$ owns $y$ in $s_{2}$, there is a situation $s_{3}$ such that $s_{3} \leq s_{1}$ and $s_{3}$ is a minimal situation such that $s_{2} \leq s_{3}$ and the unique farmer in $s_{3}$ beats in $s_{3}$ the unique donkey in $s_{3}$ and there is an individual $z$ such that $z$ is a priest in $s_{3}$ and the unique priest in $s_{3}$ beats in $s_{3}$ the unique donkey in $s_{3}$,

where $s_{1}$ is the speech situation and $s_{2}$ and $s_{3}$ are minimal situations consisting in the individuals and relations specified.

Elbourne is not concerned with sentences such as (1), but we could use (7) to get an idea of how (1) would be represented in his system. The representation of (1) would be something along the following lines:

(8) $\lambda s_{1}$. for every minimal situation $s_{2}$ such that $s_{2} \leq s_{1}$ and there is an individual $x$ such that $x$ is John in $s_{2}$ and there is an individual $y$ such that $y$ is a cigarette in $s_{2}$ and $x$ smokes $y$ in $s_{2}$, there is a situation $s_{3}$ such that $s_{3} \leq s_{1}$ and $s_{3}$ is a minimal situation such that $s_{2} \leq s_{3}$ and it rains in $s_{3}$,

11 See Kratzer (2014) for an overview. 
where $s_{1}$ is the speech situation and $s_{2}$ and $s_{3}$ are minimal situations consisting in the individuals and relations specified.

This treatment of (1) avoids the conclusion of the Binding Argument in much the same way in which Cappelen and Hawthorne's analysis does. If (8) is the representation of (1) in the situation framework, then, again, (IC) doesn't hold - there is no variable for locations in (8). But if it doesn't hold, then it means that either (IC) doesn't follow from the premises, and thus the argument is not valid, or one of the premises needed for its derivation is rejected. Again, both are the case. Premise 3 remains ambiguous between a reading according to which there is no binding without a bindable variable in the logical form simpliciter, and a reading according to which there is no binding without a bindable variable of the same kind as the bound parameter (here, location) in the logical form. On the first of these readings, premise 3 holds under the current analysis (since we have a variable for situations in (8)), but (IC) doesn't follow; on the second reading, the premise is rejected (since there is no location variable in the logical form of (1)), but the location of rain is nevertheless bound. As before, this means that in neither interpretation the intermediary conclusion (IC) can be derived, and thus that the Binding Argument is blocked.

\subsection{Quantifying over contexts}

A different way to account for the bound reading of (1) is to replace variables for locations in the object language with variables over more encompassing entities in the metalanguage. The first of the two views found in the literature is Pagin's (2005), consisting in an appeal to quantification over contexts. Contexts, for Pagin, will be modeled as "sequences of context elements, or equivalently as assignments of values to a set of context parameters. For any context $\mathrm{c}$, the associated model will contain as elements, to begin with, the speaker of c, if any, the addressee, if any, the (contextually salient) time of c, the location", and, in general, "anything over and above the sentence (actually or potentially) uttered that is available and may be employed for achieving communicative success" (Pagin 2005: 328). Sentences will be true relative only to contexts, or indices, as in Montagovian semantics. Variables for contexts are variables in the metalanguage, and can be bound by quantifiers in the metalanguage. Such binding in the metalanguage is useful in order to handle quantifiers such as 'every time' that bind context elements - in this case, time. Using this idea, a sentence containing such a quantifier could be represented as

(9) 'Every time'^ $s$ is true at $c$ if for every context $c, s$ is true at $\mathrm{T}(c)$,

where ${ }^{\wedge}$ is the concatenation sign, $s$ is a sentence, $c$ the utterance context and $\mathrm{T}(c)$ is the time of $c$. But the original sentence 'every time'^ $s$ could be context sensitive, 
and thus will be itself evaluated relative to a context. To capture this situation, 'every time' will be construed as quantifying over contexts $c$ ' which differ from $c$ with respect to time but not in other respects. 'Every time`^ $s$ will thus be represented as

(10) 'Every time'^ $s$ is true at $c$ if for every context $c$ ' $\approx c / t, s$ is true at $c$ ',

where ' $c$ ' $\approx c / t$ ' is to be read as 'context $c$ ' differs from context $c$ at most with respect to time". Now, when it comes to sentences such as (1), in order to account for the bound reading of that sentence we need first to let location vary together with time in the clause for 'Every time'^ $s$. This is accomplished in (11):

(11) 'Every time'^ $s$ is true at $c$ if for every context $c$ ' $\approx c / t+l, s$ is true at $c$ ',

where ' $c$ ' $\approx c / t+l$ ' is to be read as 'context $c$ ' differs from context $c$ at most with respect to time and location". Assuming that (1) is interpreted as a quantified conditional of the form

(12) 'Every time’^ 'if John lights a cigarette, then it rains'

and substituting the new rendering of (1) for $s$ in (11), we get

(13) 'Every time'^ 'if John lights a cigarette, then it rains' is true at $c$ if for every context $c^{\prime} \approx c / t+l$, 'if John lights a cigarette, then it rains' is true at $c$ '.

Now, if we assume that the following holds:

(14) 'if John lights a cigarette, then it rains' is true at $c$ ' if (if John lights a cigarette at $\mathrm{T}\left(c^{\prime}\right)$ at $\mathrm{L}\left(c^{\prime}\right)$, then it rains at $\mathrm{T}\left(c^{\prime}\right)$ at $\left.\mathrm{L}\left(c^{\prime}\right)\right)$,

where $\mathrm{T}\left(c^{\prime}\right)$ is the time of $c^{\prime}$ and $\mathrm{L}\left(c^{\prime}\right)$ is the location of $c^{\prime}$, we finally get (15) as the ultimate representation of (1) in Pagin's framework:

(15) 'Every time'^ 'if John lights a cigarette, then it rains' is true in $c$ if for every context $c^{\prime} \approx c / t+l$, if John lights a cigarette at $\mathrm{T}\left(c^{\prime}\right)$ at $\mathrm{L}\left(c^{\prime}\right)$, then it rains at $\mathrm{T}\left(c^{\prime}\right)$ at $\mathrm{L}\left(c^{\prime}\right)$

which gives us the desired reading.

In Pagin's account, the conclusion of the Binding Argument is avoided by rejecting premise 3 . We start by noting that, although we find a variable for locations in (15) - L $\left(c^{\prime}\right)$ - it is a variable belonging to the metalanguage and not to the object language. This makes (IC) fail. If we ask again why this happens, we are faced with the same options as before. And, again, we find that premise 3 is ambiguous between the two readings mentioned above. This time, however, premise 3 fails in both of its readings because according to Pagin's account there simply is no variable of the relevant kind in (15). We have thus a more direct blocking of the Binding Argument by straightforwardly rejecting premise 3. 


\subsection{Quantifying over indices}

The second way of accounting for the bound reading of (1) by appeal to quantification over entities in the metalanguage has been developed by Lasersohn (2008). His proposal is to "quantify directly over the [relevant] index, setting and resetting its value in tandem with the variable introduced by a quantifier" (2008: 324). Lasersohn is primarily interested in bound cases of predicates of personal taste, but he applies the same solution to locations as well.

The first thing to mention about Lasersohn's view is that it is a relativist view about locations. Thus, one main tenet of his view is that the circumstances of evaluation (the index, in Lasersohn's Lewisian jargon) contain a location parameter. For Lasersohn, sentences will be true relative to a model, an assignment, a context and an index - index which comprises, besides possible worlds and other unorthodox parameters ${ }^{12}$, a location parameter as well, so that a sentence is true in a context if and only if the proposition expressed by the sentence in that context is true relative to the world of the context and the location of the context (or, more precisely, the location of the speaker in the context). Now, Lasersohn's main claim is that quantifiers are able to bind both variables in the object language and parameters in the index (which are variables in the metalanguage). A certain quantifier, such as 'every reporter' (see example (18) below) could bind both a variable for individuals in the object language and a certain parameter in the index - say, the location parameter. For Lasersohn, quantifiers are sentence-abstract forming operators: when they bind variables in the object language, their effect could be described as in (16); when they bind variables in the metalanguage - specifically the location parameter - their effect could be described as in (17):

$$
\begin{aligned}
& {[[\lambda n \varphi]]^{M, g, c, w, l}=\left\{x \in U \mid[[\varphi]]^{M, g[x / n], c, w, l, l}=1\right\}} \\
& {[[\pi n \varphi]]^{M, g, c, w, l}=\left\{x \in U \mid[[\varphi]]^{M, g[x / n], c, w, L(x)}=1\right\},{ }^{13}}
\end{aligned}
$$

where $\varphi$ is a sentence, $M$ is a model, $g$ an assignment function, $c$ a context, $w$ a possible world, $l$ a location, $g[x / n]$ is that sequence in which $x$ is the $n$-th

12 Besides possible worlds, Lasersohn includes a parameter for judges in the index. And, to be more precise, in his view the location parameter is introduced in the index via another parameter he calls "the perspective point", which is supposed to take care of all shiftable perspectival features of contexts related to the speaker, such as location and time. Since location is the only relevant parameter here, I present the view as if location were the only unorthodox parameter of the index. 13 I have slightly modified Lasersohn's clauses to align with the simplification made (see previous footnote). For Lasersohn's original clauses of the two operators, see Lasersohn (2008: 313 and 331, respectively). 
element and which agrees with $g$ in all other positions, $L(x)$ is the location of $x$, $\mathrm{U}$ the set of individuals and $\lambda$ and $\pi$ are the two sentence-abstract forming operators. As (16) and (17) show, $\lambda$ manipulates the assignment function (lambdaabstraction), whereas $\pi$ manipulates the location parameter of the index, so that the truth of $\varphi$ is dependent on the values taken by $L(x)$.

In order to deal with bound readings of sentences containing locational expressions without having to postulate variables for locations in the object language, Lasersohn allows quantifiers to bind parameters in the index. To use Lasersohn's own example, sentence

(18) Every reporter visited a local bar,

has a reading according to which every reporter visited a bar that is local relative to the location of each reporter that visited the bar. This bound reading of (18) is usually accounted for by postulating a variable for locations in the logical form of 'local', an object language variable that could be free or bound. But this does not need to happen in Lasersohn's account. (18) will be represented in Lasersohn's system as

(19) $\left[[\right.$ every reporter $] \pi_{1}\left[\left[\right.\right.$ some bar that $\lambda_{2}\left[\right.$ pro $_{2}$ is-local $\left.]\right] \lambda_{3}\left[\right.$ pro visited pro $\left.\left.\left._{3}\right]\right]\right]$,

where the quantifier 'every reporter' binds both a variable for individuals in the object- language ( $p r \theta_{1}$ ) and the location parameter in the index. But the important point to note is that 'every reporter' doesn't bind any object language variable for locations, since, as can be seen in (19), 'local' doesn't have a variable for locations to be bound.

Lasersohn doesn't tell us how sentence (1) would be represented in his framework, but we get an idea from (19) how this could be done. Here is one attempt at representing (1) using Lasersohn's apparatus:

(20) [[every time] $\lambda_{1}$ [John lights a cigarette at pro 1$\left.]\right] \pi_{2}$ [it rains at pro 1$\left.]\right]$.

In (20), the quantifier 'every time' binds a variable for times in the object language $\left(\right.$ pro $\left._{1}\right)$, whereas the more complex quantifier 'every time John lights a cigarette', containing 'every time' as its part, binds the location parameter in the index. But the important point to note is, again, that 'every time John lights a cigarette' doesn't bind any object language variable for locations, since, as can be seen in (20), 'rain' doesn't have a variable for locations to be bound.

Thus, in Lasersohn's account, as well as in Pagin's, the conclusion of the Binding Argument is avoided by rejecting premise 3. We start by noting that there is a variable for location in the clause for the evaluation of (1) given in (17) - that is, $\mathrm{L}(x)$; however, it is a variable belonging to the metalanguage and not to the object language. This makes (IC) fail. If we ask again why this happens, we are 
faced with the same options as before. And, again, we come to the conclusion that premise 3 is ambiguous between the two by now familiar readings. This time, however, premise 3 fails in both of its readings because according to Lasersohn's account there simply is no variable of the relevant kind in (20). Here we also have a more direct blocking of the Binding Argument by straightforwardly rejecting premise 3.

\subsection{The variadic operators approach}

Finally, another alternative way to account for the bound readings of sentences like (1) without postulating variables for locations in the target sentences comes from Recanati, the chief opponent to the Binding Argument for locations. The main feature of Recanati's view is his appeal to "variadic functions" - functions that take as input a predicate with a certain number of arguments and yield a new, related predicate with one more (or less) arguments. In the case of the "expansive" variadic function (the one that creates a new predicate with an additional argument), the new argument stands for what Recanati calls a "circumstance": "a time, a location, a manner, or what not” (Recanati 2002: 319).

Variadic functions are represented by variadic operators. The effect of the expansive variadic operator on a predicate can be captured by the following equivalence:

(21) $\mathbf{V}\left(\lambda x_{1} \ldots \lambda x_{n} \cdot \varphi\left(x_{1} \ldots x_{n}\right)\right) \equiv \lambda x_{1} \ldots \lambda x_{n} \cdot \lambda y \cdot \varphi^{\star}\left(x_{1} \ldots x_{n}, y\right)$,

where $\mathbf{V}$ is the expansive variadic operator, $\lambda x_{1} \ldots \lambda x_{n} \cdot \varphi\left(x_{1} \ldots \mathrm{x}_{n}\right)$ is the input predicate with $n$ arguments (here represented as unsaturated), $\lambda x_{1} \ldots \lambda x_{n^{*}} \lambda_{y^{*}} \varphi^{\star}\left(x_{1}\right.$ $\left.\ldots x_{n}, y\right)$ is the new predicate with $n+1$ arguments and $y$ is the newly introduced variable.

Now, following other authors with similar proposals, Recanati makes the claim that several natural language expressions can be handled by using the variadic operator just defined. ${ }^{14}$ Here is what Recanati says about the category of modifiers: "modifiers are syntactically optional. They make a predicate out of a predicate. If we start with a simple predicate, say 'rain', we can make a different predicate out of it by adjoining an adverb such as 'heavily' or a prepositional phrase such as 'in Paris'. (...) Semantically, I suggest that we construe the modifier as contributing a certain sort of [operator] which I call a variadic [operator]." (2002: 319). The contribution of the variadic operator won't be the only one made by such expressions, however. In fact, they will be treated as having a twofold

14 Such as McConnell-Ginet (1982) and Keenan and Faltz (1985). 
semantic effect: on one hand, they create a new, related predicate that differs from the input predicate by having an additional argument place occupied by a variable of a certain sort (the effect of the variadic operator); on the other hand, they provide a value for that variable.

Let us apply this apparatus to the case at hand. With $\mathbf{V}_{\boldsymbol{x}: \boldsymbol{y}}$ capturing the effect of the modifier, subscript $x$ indicating the type of variable the operator introduces and subscript $\boldsymbol{y}$ indicating the value given to that variable, sentence

(22) It is raining in Paris

will be represented as

(23) V location: Paris (rain),

which is equivalent with

(24) rain_in (Paris).

We see that the locational expression 'in Paris' is treated as contributing both a variadic operator which transforms the zero-place predicate 'rain' into a new predicate with an additional argument place for locations (symbolized in (24) as 'rain_in') and the specific value for that extra argument place of the newlycreated predicate (in this case, Paris).

What about sentence (1), our main target? Recanati's claim is that, as with prepositional phrases like 'in Paris', quantifier phrases like 'every time John lights a cigarette' also contribute a variadic operator. Simplifying greatly, (1) will be represented using the variadic operators apparatus as

(25) $\forall l$ (every time John lights a cigarette in $l \rightarrow \mathbf{V}_{\text {location: } l}$ (rain))

which is equivalent with

(26) $\forall l$ (every time John lights a cigarette in $l \rightarrow$ rain_in $(l)$ ).

We see that the quantifier phrase 'every time John lights a cigarette' is treated as both contributing a variadic operator which transforms the zero-place predicate 'rain' into a new predicate with an additional argument place for locations and binding the location variable of the newly-created predicate. The difference between prepositional phrases like 'in Paris' and quantifier phrases like 'every time John lights a cigarette' thus lays in the fact that the first provides specific value for the location variable, while the second simply binds that variable.

How does this treatment avoid the conclusion of the Binding Argument? The main difference between this approach and the ones investigated before is that according to the present one, the first three premises, the intermediary conclusion (IC) and the bridging principle (BP) all hold, but conclusion 4 doesn't 
follow. This is because, even if there is a variable for locations in $Q(s)$, there is none in $s$ taken in isolation. The reason for that is, of course, the presence of the variadic operator, which is responsible for the introduction of the location variable in $Q(s)$.

Now, Recanati has used the apparatus of variadic operators within a truthconditional pragmatic theory that makes essential appeal to optional pragmatic processes like free enrichment. In connection with (1), for example, Recanati claims that the variadic operator is contributed precisely by such a process. Many authors have expressed concern with respect to the nature of Recanati's optional pragmatic processes, the most widespread worry being that they lack the systematicity and the constrained character of mandatory processes like saturation. ${ }^{15}$ But it should be clear that the variadic operators apparatus itself caries no commitment to optional (or of any kind, for that matter) pragmatic processes. Thus, one could claim that the process by which the variadic operator is contributed, although still pragmatic in nature, is more constrained than free enrichment. Alternatively, one could claim, as done here, that the variadic operator is part of the logical form of (1). Regardless of which of these alternatives is ultimately adopted, the result that the conclusion of the Binding Argument is avoided by giving (1) a variadic operator treatment remains.

\section{Conclusion}

The Binding Argument has received its fair share of criticism in the literature. In this paper I focused on a weaker interpretation of the argument according to which the postulation of variables for locations in the logical form of meteorological sentences such as "It is raining" is the best available explanation of binding phenomena such sentences give rise to. Such an interpretation, it seems to me, is compatible with criticisms like those put forward by Cappelen and Lepore (2002) or by Pupa and Troseth (2011) that best apply to the other, stronger interpretations of the argument.

However, this doesn't mean that the Binding Argument in its third interpretation can be freely used: in fact, my aim was to show precisely the contrary. Thus, in section 3 I showcased a number of alternative ways to account for the binding data that have motivated the Binding Argument. The fact that such ways have

15 The most vivid challenge appears in Stanley (2007, chapter 6). For replies to such an allegation, see Elbourne (2008), Hall (2008) or Recanati (2010). 
been proposed is, of course, not an indication of their correctness. However, what is important in this context is not so much whether they are ultimately correct, but whether they have been ruled out as incorrect by the proponents of the Binding Argument in its third interpretation. So far, this is something that hasn't happened. To be fair, some of the views presented above have been criticized. For example, Stanley (2007, chapter 6) has argued that Pagin's (2005) view is problematic with respect to certain quantified sentences. But Pagin's view is just one alternative way to account for the binding data. And although Recanati's truthconditional pragmatic view has been the focus of repeated criticisms by Stanley and other truth-conditional semanticists, the apparatus of variadic operators in itself has been rarely addressed - despite its importance in connection to the Binding Argument being acknowledged (Stanley 2007, chapter 7). ${ }^{16}$ This is not to say, of course, that the views that have not been engaged with in the literature are problem-free. ${ }^{17}$ But, for the Binding Argument in its third interpretation to work, what is needed is a more thorough investigation of all the alternatives on the market and a careful comparison of their respective virtues and shortcomings with those of the view postulating variables for locations in the logical form. It might be that at the end of such an enterprise the postulation of variables for locations in the logical form of sentences such as 'It is raining' is our best choice. However, as things stand now, no such claim can be reasonably made.

Although I have mentioned some criticisms of the Binding Argument in its first and second interpretation, my focus in this paper has been on the third. But if one accepts that those criticisms are on the right track, the Binding Argument retains very little from its (once considered great) methodological power. Since the Binding Argument has mainly been employed in support of truth-conditional semantic views about "It is raining" and other types of sentences, it follows that the argument cannot be used by the proponents of such views. To be sure, this is not something that I have shown in this paper. What I did show, however, is that if one settles for the argument's third interpretation, it cannot be used to support a truth-conditional semantic view about "It is raining" and its ilk. ${ }^{18}$

16 This is, of course, not to accuse Stanley of any omissions - most of the alternative views to his own have been put forward after his writings on the Binding Argument.

17 For some problems with all the views presented above, except the variadic operators approach, see Zeman (2011, chapter 3).

18 I'm grateful to Isidora Stojanovic for giving me the possibility of contributing to this volume and to Sarah-Jane Conrad for her support and feedback, as well as to an anonymous reviewer for this volume. Research for this paper has been financially supported by a MINECO Juan de la Cierva grant (JCI-2012-12974) and by the Semantic Content and Conversational Dynamics project (FFI2012-37658) at the University of Barcelona. 


\section{References}

Breheny, Richard. 2003. On bindability. Amsterdam Colloquium 14. 81-87.

Cappelen, Herman \& Ernie Lepore. 2002. Indexicality, binding, anaphora and a priori truth. Analysis 62.4. 271-281.

Cappelen, Herman \& John Hawthorne. 2007. Locations and binding. Analysis 67(2). 95-105. Carston, Robyn. 2002. Thoughts and utterances: The pragmatics of explicit communication. Oxford: Blackwell Publishing.

Chomsky, Noam. 1957. Syntactic Structures. The Hague: Mouton.

Cohen, Jonathan \& Samuel C. Rickless. 2007. Binding arguments and hidden variables. Analysis 67(1). 65-71.

Collins, John. 2007. Syntax, more or less. Mind 116(464). 805-850.

Davidson, Donald. 1967. The logical form of action sentences. In Nicholas Rescher (ed.), The logic of decision and action 81-95. Pittsburgh: University of Pittsburgh Press.

Elbourne, Paul. 2005. Situations and Individuals. Cambridge MA: MIT Press.

Elbourne, Paul. 2008. The argument from binding. Philosophical Perspectives 22(1). 89-110. Hall, Alison. 2008. Free enrichment or hidden indexicals? Mind \& Language 23(4). 426-456. Jacobson, Pauline. 1999. Towards a variable-free semantics. Linguistics and Philosophy 22(2). 117-184.

Kaplan, David. 1989. Demonstratives. In Joseph Almog, John Perry \& Howard Wettstein (eds.), Themes from Kaplan, 481-563. Oxford: Oxford University Press.

Keenan, Edward L. \& Leonard M. Faltz. 1985. Boolean semantics for natural language. Dordrecht: D. Reidel.

Kratzer, Angelika. 2014. Situations in natural language semantics. The Stanford encyclopedia of philosophy (Spring 2014 Edition), Edward N. Zalta (ed.). http://plato.stanford.edu/ archives/spr2014/entries/situations-semantics/_ (accessed 01.09.2015).

Lasersohn, Peter. 2008. Quantification and perspective in relativist semantics. Philosophical Perspectives 22(1). 305-337.

Lewis, David. 1980. Index, context, and content. In Stig Kanger \& Sven Öhman (eds.), Philosophy and grammar, 79-100. Dordrecht: D. Reidel.

McConnell-Ginet, Sally. 1982. Adverbs and logical form: A linguistically realistic theory. Language 58. 144-184.

Neale, Stephen. 2007. On location. In Michael O'Rourke \& Corey Washington (eds.), Situating semantics. Essays on the philosophy of John Perry, 251-394. Cambridge, MA: MIT Press.

Pagin, Peter. 2005. Compositionality and context. In Gerhard Preyer \& Georg Peter (eds.), Contextualism in philosophy: Knowledge, meaning, and truth, 303-348. Oxford: Clarendon Press.

Recanati, François. 2002. Unarticulated constituents. Linguistics and Philosophy 25(3). 299-345.

Recanati, François. 2004. Literal meaning. Cambridge: Cambridge University Press.

Recanati, François. 2007. Perspectival thought: A plea for (moderate) relativism. Oxford University Press.

Recanati, François. 2010. Truth-Conditional Pragmatics. Oxford: Clarendon Press.

Pupa, Francesco \& Erika Troseth. 2011. Syntax and Interpretation. Mind and Language 26(2). 185-209.

Schaffer, Jonathan. 2011. Perspective in Taste Predicates and Epistemic Modals. In Andy Egan \& Brian Weatherson (eds.), Epistemic Modality, 179-226. Oxford: Oxford University Press. 
Sennet, Adam. 2008. The binding argument and pragmatic enrichment, or, why philosophers care even more than weathermen about 'raining'. Philosophy Compass 3(1). 135-157.

Stanley, Jason. 2007. Language in context. Selected essays. Oxford: Clarendon Press.

Zeman, Dan. 2011. The Semantics of implicit content. University of Barcelona dissertation. http://www.tdx.cat/bitstream/handle/10803/22693/DZ_THESIS.pdf?sequence=1.

Zeman, Dan. 2015. Relativism and bound predicates of personal taste: An answer to Schaffer's argument from binding. Dialectica 69(4). 155-183. 